\title{
Beam transfer function measurements used to probe the transverse Landau damping at the LHC
}

\author{
C. Tambasco ${ }^{*}$, T. Pieloni, and J. Barranco® \\ Particle Accelerator Physics Laboratory, Institute of Physics, EPFL, Lausanne, Switzerland \\ X. Buffat, T. Levens®, E. Métral, and B. Salvachua \\ CERN, CH-1211 Geneva 23, Switzerland
}

(Received 18 October 2019; accepted 18 June 2020; published 16 July 2020)

\begin{abstract}
The Landau damping mechanism stabilizes the impedance driven head-tail modes in hadron synchrotrons. Because of the frequent coherent beam instabilities observed in the Large Hadron Collider in 2012, the Landau damping of the beams was doubted. Therefore, a transverse beam transfer function (BTF) system was installed in order to probe the transverse Landau damping of the beams and compare the measurements with expectations. The results of the BTF measurements acquired during several experiments in the LHC are summarized including different machine configurations. The method developed for the data analysis is described, and the comparison between the models and the measurements is also presented.
\end{abstract}

DOI: 10.1103/PhysRevAccelBeams.23.071002

\section{INTRODUCTION}

At the Large Hadron Collider (LHC), the coherent instabilities driven by the beam coupling impedance are passively mitigated by the Landau damping mechanism [1]. This stabilizing mechanism is generated by the diversification of oscillation frequencies of protons as a function of their amplitude inside the beams (tune spread). Any lattice nonlinearities present in the accelerator contribute to the tune spread in the beams. In the LHC, specific magnets, known as Landau octupoles, are used in order to provide enough tune spread to stabilize the proton beams during machine operation [2]. The evaluation of the stability thresholds in the LHC is based on the computation of the stability diagram (SD) including the tune spread generated by Landau octupoles and beam-beam interactions [3]. The stability diagram can be studied experimentally via transverse beam transfer function (BTF) measurements [4], since it is given by its reciprocal $[5,6]$. The BTFs are sensitive to the tune spread in the beams as well as to particle distribution changes. Any diffusive mechanisms, caused by resonance excitations or noise, may lead to a change of the particle distribution, causing a possible lack of Landau damping of the

*claudia.tambasco@epfl.ch

Published by the American Physical Society under the terms of the Creative Commons Attribution 4.0 International license. Further distribution of this work must maintain attribution to the author(s) and the published article's title, journal citation, and DOI. impedance coherent modes previously damped by lying within the unperturbed SD. The derivation of the relationship between the SD and the BTF follows [5,7]. Each particle in the beam oscillates in the horizontal and vertical plane with a frequency $q_{x, y}\left(J_{x}, J_{y}\right)$ depending on the particle amplitude defined by the transverse actions $J_{x}$ and $J_{y}$. The ensemble of the oscillation frequencies of the particles in the transverse plane, according to their amplitude in the beams, defines the so-called transverse detuning with amplitude or tune spread. In the presence of an external excitation, as the one introduced by rigid dipole oscillations caused by the machine impedance, the particle motion can be considered as a driven oscillator under a force of frequency $\Omega$ and infinitesimal amplitude $B$, for instance, in the horizontal plane:

$$
f_{x}(t)=B_{x} e^{-i \Omega_{x} t}
$$

with $\Omega_{x}=Q_{x} \cdot \omega_{0}$, where $\omega_{0}$ is the revolution angular frequency and $Q_{x}$ is the tune value close to the amplitudedependent horizontal tune $q_{x}\left(J_{x}, J_{y}\right)$. A perturbative solution of the Vlasov equation leads to a collective beam response [7]:

$\langle x\rangle=2 \pi^{2} \omega_{0} f_{x}(t) \int_{0}^{\infty} \frac{J_{x}}{Q_{x}-q_{x, y}\left(J_{x}, J_{y}\right)} \frac{d \psi}{d J_{x}} d J_{x} d J_{y}$

with $\psi\left(J_{x}, J_{y}\right)$ the particle distribution function. The driving term in Eq. (1) can be expressed in terms of coherent tune shifts $\Delta Q_{x}^{\text {coh }}=\Delta \Omega_{x} / \omega_{0}$ induced by the machine impedance through the relation [7] 


$$
f_{x}(t)=-2 \Delta \Omega_{x}\langle x\rangle=-2 \omega_{0} \Delta Q_{x}^{\mathrm{coh}}\langle x\rangle .
$$

By substituting this last relation in Eq. (2), the following dispersion integral [7] is obtained:

$$
\mathrm{SD}_{(x, y)}^{-1}=\frac{-1}{\Delta Q_{x, y}^{\mathrm{coh}}}=\int_{0}^{\infty} \frac{J_{x, y}}{Q_{x, y}-q_{x, y}\left(J_{x}, J_{y}\right)} \frac{d \psi}{d J_{x, y}} d J_{x} d J_{y} .
$$

The same formalism has been applied in the vertical plane. The double integral in Eq. (4) is proportional to the socalled BTF that describes the response of the beam's dipole moment to a driving force [5,7]. Therefore, the BTFs are related to the SDs through the dispersion integral:

$$
\begin{gathered}
\operatorname{BTF}_{(x, y)}=A_{x, y} \int_{0}^{\infty} \frac{J_{x, y}}{Q_{x, y}-q_{x, y}\left(J_{x}, J_{y}\right)} \frac{d \psi}{d J_{x, y}} d J_{x} d J_{y}, \\
\mathrm{SD}_{(x, y)}^{-1}=\frac{-1}{\Delta Q_{x, y}^{\mathrm{coh}}}=\frac{\mathrm{BTF}_{(x, y)}}{A_{x, y}} .
\end{gathered}
$$

The SD defines an area in the complex plane, with the imaginary part $\operatorname{Im}\left(\Delta Q_{x, y}^{\text {coh }}\right)$ and the real part $\operatorname{Re}\left(\Delta Q_{x, y}^{\text {coh }}\right)$ of the coherent tune shifts on the $y$ axis and $x$ axis, respectively. In this area, the coherent tune shifts of dipole oscillations are stable in the presence of certain detuning with amplitude (tune spread) in the beams and, in particular, for $\operatorname{Im}\left(\Delta Q_{x, y}^{\text {coh }}\right)<0$ [2]. The larger the transverse tune spread in the beams, the larger the SD. The proportionality constant $A_{x, y}$ is linked to both the excitation amplitude in the corresponding plane and the beam conditions (tune spread, particle distribution). The BTFs are also powerful beam diagnostic tools, for example, for tune monitoring [8] and chromaticity measurements. They also provide information about the transverse and longitudinal impedances $[9,10]$. In the presence of colliding beams, BTFs have been also used to estimate the tune spread in the presence of coherent beam-beam modes [11]. The BTFs can detect excited coherent modes in the beams $[6,12]$ and can give information about the beam-beam coupling by measuring the cross talk between two colliding beams [13]. In the LHC, the BTF system was installed in order to give insights into Landau damping of the beams by measuring the SD and comparing with the theoretical models. Multiple coherent instabilities have been observed in the LHC since the 2012 physics run related to a loss of Landau damping, although the transverse beam stability of the beams was maximized throughout the operational cycle [14]. Therefore, the knowledge of the Landau damping of the beams was doubted, and the transverse BTF system was installed in 2015 in order to compare the measured BTF with the models used to predict the beam stability in the LHC. Measurements were acquired for several machine configurations. This article summarizes the results of the measurements acquired over several experiments with a single beam [15-20]. The method for the data analysis and the comparisons with the expectations are presented.

\section{THE TRANSVERSE BEAM TRANSFER FUNCTION SYSTEM IN THE LHC}

The development and the installation of a transverse BTF system has been carried out by the Beam Instrumentation Group. Measurements have been performed during dedicated machine development periods. During a BTF acquisition, the chosen plane of the beam is safely excited, i.e., without causing detectable losses and/or emittance blowup, within the betatron frequency range of interest. A periodic excitation at a frequency close to the tune is applied to the beam, and the amplitude and phase (with respect to the external excitation) of the resulting transverse coherent oscillation are detected by a beam position monitor. Specifically, the beam is excited by the kickers of the transverse damper (called ADT) at a certain tune $Q$, while the beam response is recorded by the beam position monitors (BPMs) of the base band (BBQ) measurement system [21]. A typical BTF measurement is shown in Fig. 1 for beam 1 (B1) in the horizontal plane at injection energy. The amplitude response of the beam is the blue line, while the phase response of the beam is the red line. The maximum peak in the amplitude corresponds to the fractional part of the horizontal betatron coherent tune $\left(q_{x} \approx 0.284\right)$. The first synchrotron sidebands are also visible in the amplitude response occurring at $q_{x} \pm q_{s}$, where $q_{s}=5 \times 10^{-3}$ is the fractional longitudinal tune at injection energy. The corresponding jumps are visible in the phase response. The synchrotron sidebands are visible due to nonzero chromaticity $Q^{\prime} \approx 5.0$ during the measurement
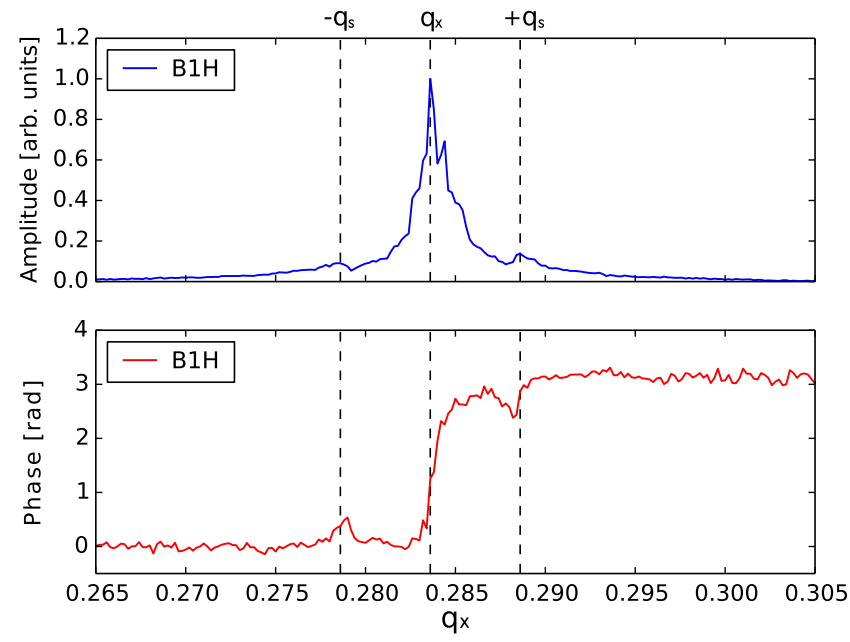

FIG. 1. Example of a BTF measurement: amplitude and phase response for B1 at the LHC injection energy. Synchrotron sidebands are visible in the amplitude, with the corresponding phase jumps at $q_{x} \pm q_{s}$ (where $\left.q_{s}=5 \times 10^{-3}\right)$. 
acquisitions. The phase response varies from 0 to $\pi$ during the excitation, with a value of $\pi / 2$ at the coherent tune.

\section{A. The fitting method}

The amplitude of the beam oscillation driven by the BTF excitations and detected by the BPMs of the BBQ system is not calibrated, meaning that the excitation amplitudes cannot be expressed in terms of the beam position. Therefore, the amplitude response of the beam can be expressed in arbitrary units only. Because of the nonlinearity of the BBQ detectors, the calibration of the amplitude response of the system is complex and is dependent on the beam parameters themselves. Because of the limited time available for dedicated studies, this procedure has not been yet performed in the LHC. In addition, a full calibration of the system cannot be accomplished, since the proportionality constant $A_{x, y}$ in Eq. (5) cannot be known a priori. Indeed, it depends not only on the calibration factor of the BTF excitation amplitude, but also on the conditions of the beam itself, specifically on the tune spread introduced by the machine nonlinearities during the measurements. For a fixed excitation amplitude and a given normalized beam emittance, the maximum height of the BTF amplitude response (occurring at the coherent tune) decreases by increasing the tune spread in the beam. Figure 2 shows the maximum height of the simulated BTF amplitude response [the blue dotted line in Fig. 2(a)] as a function of the octupole strength (indicated in the picture as the LHC octupole current). The BTF simulations have been carried out by using the COMBI code [22-24]. The code allows one to track the particles in a simplified lattice, where several actions are defined such as [25] linear 6D transfer, impedance, linear detuning, etc. The COMBI code provides the evolution of the beam parameters treating the beam motion in a self-consistent way including a coherent dynamics of the beam described by as an ensemble of macroparticles. The BTF routine implemented in COMBI applies an harmonic excitation to the beam particles as a transverse kick $\left(x^{\prime}\right.$ or $\left.y^{\prime}\right)$ for the frequency range of interest over the selected number of turns $N$ for each betatron frequency step:

$$
\begin{aligned}
& x^{\prime}=A_{x}^{\mathrm{exc}} \cdot \sigma_{x}^{\prime} \sin \left(2 \pi Q_{b}\right), \\
& y^{\prime}=A_{y}^{\mathrm{exc}} \cdot \sigma_{y}^{\prime} \sin \left(2 \pi Q_{b}\right),
\end{aligned}
$$

where $A_{x, y}^{\text {exc }}$ is the excitation amplitude in the chosen plane in units of the beam divergences $\sigma_{x}^{\prime}$ and $\sigma_{y}^{\prime}$ and $Q_{b}$ is the excitation frequency. The amplitude and the phase of the BTF [Eq. (5)] are computed in the postprocessing by using the Fourier sums, for which the real and imaginary parts are (considering an horizontal BTF), respectively,

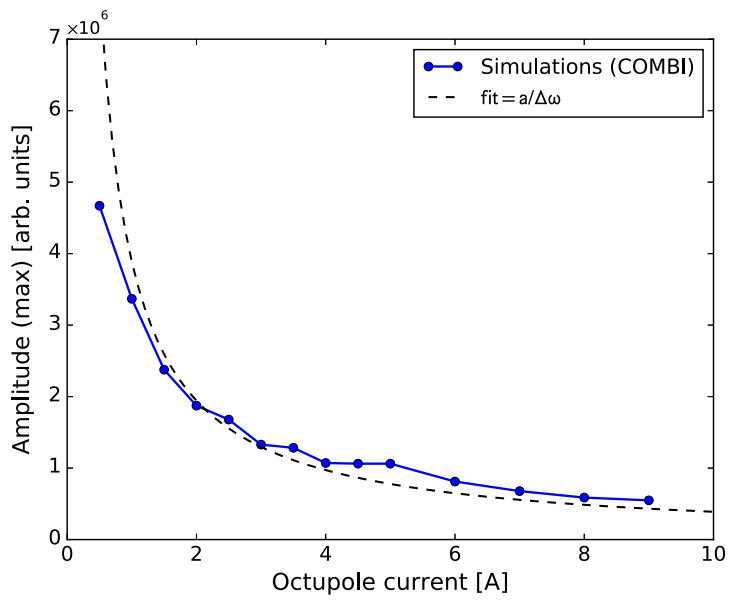

(a) Maximum height of the simulated BTF amplitude response as a function of the octupole current.

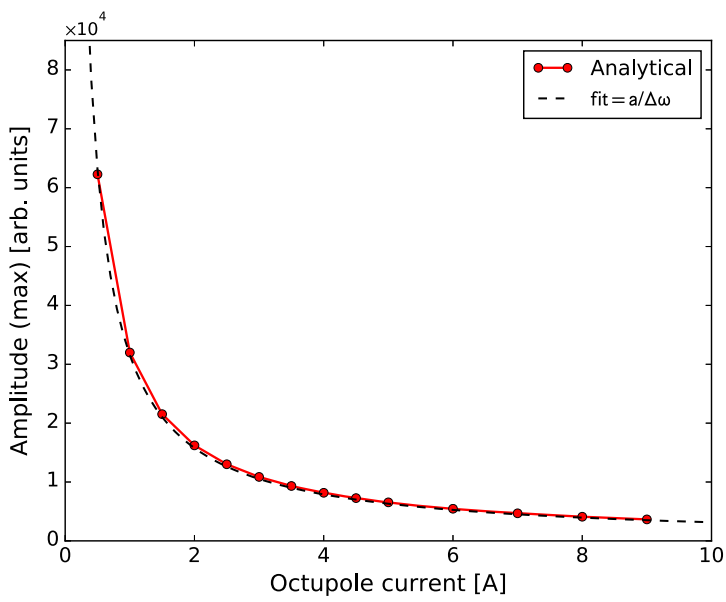

(b) Maximum height of the amplitude of the dispersion integral as a function of the octupole current.

FIG. 2. Maximum height of the BTF amplitude response simulated by using the COMBI code (the blue dotted line in (a)) and maximum height of the amplitude of the dispersion integral, evaluated analytically by using the PysSD code (the red dotted line in (b)), as a function of the octupole strength (indicated in the picture as the LHC octupole current).

$$
\begin{aligned}
& \operatorname{Re}(\mathrm{BTF})=\sum_{i=1}^{N} x_{i} \cos \left[2 \pi Q_{b} \cdot(i-1)\right], \\
& \operatorname{Im}(\mathrm{BTF})=\sum_{i-1}^{N} x_{i} \sin \left[2 \pi Q_{b} \cdot(i-1)\right],
\end{aligned}
$$

where $x_{i}$ is the transverse coordinate of the beam centroid at the $i$ th turn and the sum extends over the total number of turns $N$ during which the excitation is applied.

In the presence of a linear detuning with amplitude, i.e., with a linear dependency on the transverse actions $J_{y}$ and $J_{y}$, as the one provided by Landau octupoles [2]: 
$\Delta Q_{x}=\left[\frac{3}{8 \pi} \int \beta_{x}^{2} \frac{O_{3}}{B \rho} \mathrm{ds}\right] J_{x}-\left[\frac{3}{8 \pi} \int 2 \beta_{x} \beta_{y} \frac{O_{3}}{B \rho} \mathrm{ds}\right] J_{y}$,

$\Delta Q_{y}=\left[\frac{3}{8 \pi} \int \beta_{x}^{2} \frac{O_{3}}{B \rho} \mathrm{ds}\right] J_{y}-\left[\frac{3}{8 \pi} \int 2 \beta_{x} \beta_{y} \frac{O_{3}}{B \rho} \mathrm{ds}\right] J_{x}$,

where $O_{3}$ is the octupolar strength. The average of $\left\langle J_{x, y}\right\rangle$ is defined as the beam emittance $\epsilon_{x, y}$. Therefore, the detuning with amplitude given by the octupole magnets can be rewritten as [2]

$$
\begin{aligned}
& \Delta Q_{x}=a_{x} J_{x}+b_{y} J_{y}, \\
& \Delta Q_{y}=a_{y} J_{y}+b_{x} J_{x} .
\end{aligned}
$$

The maximum height of the BTF amplitude response decreases as $1 / \Delta q$ as shown in Fig. 2. The behavior of the simulated BTF amplitude response [Fig. 2(a)] well reproduces the fitting function $1 / \Delta q$ (dashed black line). The same trend is confirmed by the maximum height of the amplitude of the dispersion integral, evaluated analytically by using the PySSD code [3] [the red line in Fig. 2(b)], for the same case of a linear detuning with amplitude. Therefore, the amplitude response of the beam can be expressed only in arbitrary scale in the presence of an unknown tune spread in the beams. Because of this circumstance, the reconstructed stability diagram from the measurements does not reflect the correct size in the complex plane of the head-tail coherent tune shifts [3,7]. In order to overcome this problem and evaluate the measured tune spread for comparisons with expectations, the experimental data will be analyzed by using the following fitting method. The fitting function takes as input the amplitude $A_{\text {model }}\left(Q_{\text {model }}\right)$ and the phase $\varphi_{\text {model }}\left(Q_{\text {model }}\right)$ of the analytical dispersion integral computed by using the PySSD code [3] and fits the measurements to the expectations by using the following parameterization:

$\varphi\left(Q_{\text {meas }}\right)=\varphi_{\text {model }}\left[\phi_{\text {shift }}+\phi_{\text {scale }} \cdot\left(Q_{\text {model }}-Q_{0}\right)\right]$,

$A\left(Q_{\text {meas }}\right)=A_{\text {model }}\left(Q_{\text {model }}\right) \cdot A_{\text {scale }} / \phi_{\text {scale }}$,

where $Q_{\text {meas }} \approx \phi_{\text {shift }}+\phi_{\text {scale }} \cdot\left(Q_{\text {model }}-Q_{0}\right)$. Both $A_{\text {model }}$ and $\varphi_{\text {model }}$ depend on the tune spread included in the model $\left(Q_{\text {model }}\right)$, and $Q_{0}$ is the value of the transverse coherent tune in the model. The parameters provided by the result of the fit of the BTF measurements with the analytical dispersion integral of Eq. (4) are $\phi_{\text {shift }}, \phi_{\text {scale }}$, and $A_{\text {scale. }}$ The phase shift $\phi_{\text {shift }}$ relates to a change of the machine tune with respect to the one assumed in the model and does not impact the estimation of the SD. The phase scale $\phi_{\text {scale }}$ yields the ratio between the measured and modeled SD, independently of the amplitude $A_{\text {scale }}$. It is therefore an absolute measurement of the SD, under the assumption that the real amplitude detuning is only scaled with respect to the modeled one [Eq. (10)]. For example, if the phase scale $\phi_{\text {scale }}<1$, it means that the measured tune spread is smaller than the one included in the model to fit the measurements. When the BTF excitation is sufficiently small to assume that the beam distributions remains Gaussian and the amplitude detuning is dominated by the one provided by the octupole magnets, the phase scale is a linear factor as a function of the octupole strength. It is important to underline that the phase scale does not depend on the amplitude response; therefore, it is not related with the unknown calibration factor of the BTF amplitude. The phase scale is independently calculated from the slope of the measured phase response given by the fitting function $\varphi\left(Q_{\text {meas }}\right)$. The factor $A_{\text {scale }}$ is a scaling factor of the amplitude response with respect to the reference case $A_{\text {model }}\left(Q_{\text {model }}\right)$. This parameter contains the missing calibration factor due to the beam tune spread and the BTF excitation itself, and it is irrelevant in the determination of the phase scale parameter related with the measured tune spread.

Such a parameterization allows one to correlate the measured amplitude and phase to a known case and compare the measurements with the analytical models currently used to predict the transverse beam stability in the LHC. This parameterization provides a method of overcoming the unknown units of the amplitude response at the cost of relying on an approximation of the tune spread and of the beam distribution, considered in the model as a Gaussian particle distribution. In order to validate the fitting method, it has been applied to the simulated BTF response by using the COMBI code including a known linear detuning with amplitude for a Gaussian beam. The same linear detuning has been used for the evaluation of the dispersion integral used to fit the simulated data with the PySSD code. Figure 3 shows the simulated BTF response (red line) and the results of the fitting function (black line). The fit
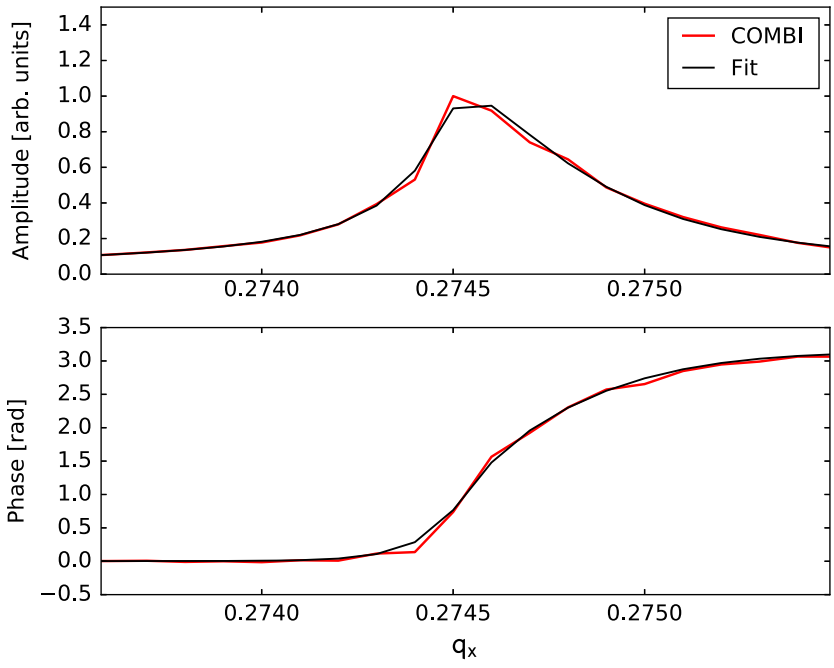

FIG. 3. Amplitude and phase BTF response simulated by using the COMBI code (red line) in the presence of linear detuning with amplitude. The black line represents the results of the parametric fit. The same tune spread has been applied for both cases, and, as expected, the fit gives a phase scale close to $1\left(\phi_{\text {scale }}=1.02\right)$. 

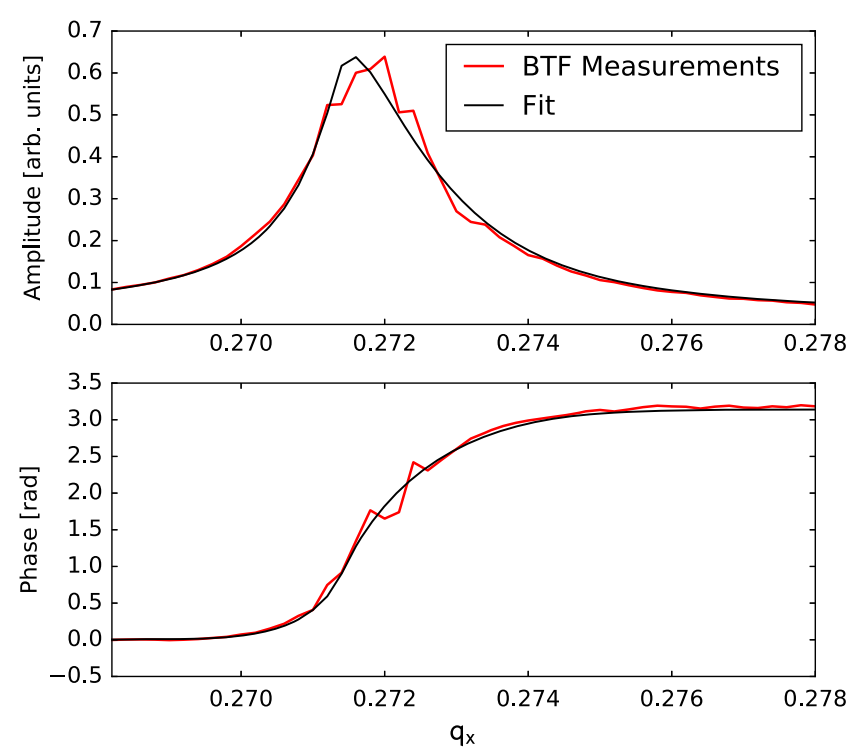

FIG. 4. Amplitude and phase response for beam 1 at the LHC injection energy (octupole current of $10 \mathrm{~A}$ ). The red line shows the BTF measurements, while the black line shows the applied fitting method using the parametrization.

reproduces well the simulated BTF response for a wellknown case, and, as expected, the phase scale results to be almost one $\left(\phi_{\text {scale }}=1.02\right)$, indicating that the expected tune spread and the fitted one from the simulation data are in agreement. The tune shift, given by the parameter $\phi_{\text {shift }}$, results to be almost zero (of the order of $1 \times 10^{-6}$ ), as expected, since the same tune has been used for both the simulations and the model. An application of the fitting method in order to reconstruct the SD from BTF measurements is shown in Fig. 4. The measurements have been

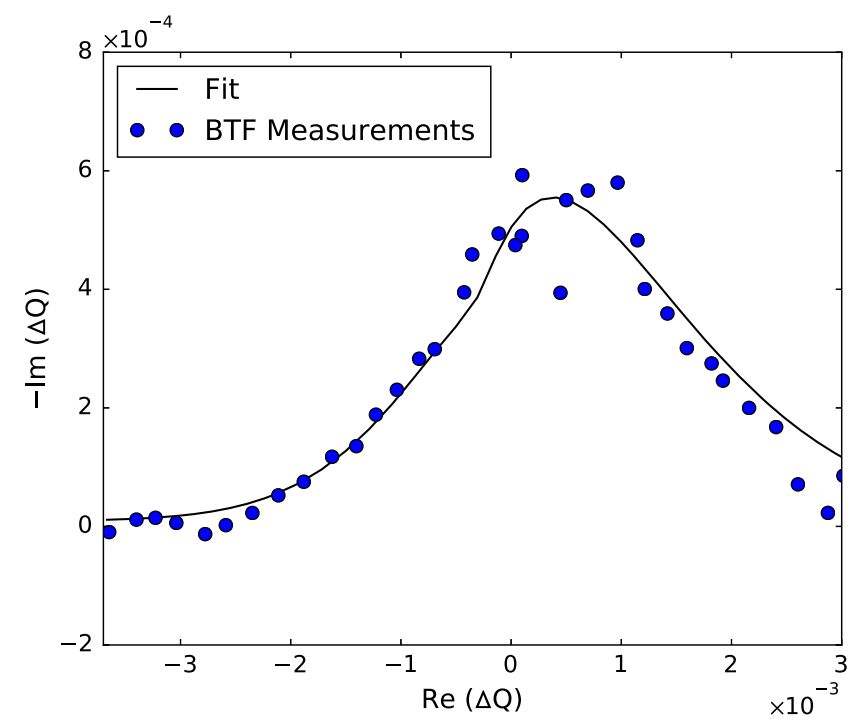

FIG. 5. Reconstructed stability diagram by using the fitting method. The blue dots are the measurements, while the black line is the corresponding SD fit given by Eq. (11). acquired at injection energy on a single bunch of beam 1 in the horizontal plane with the Landau octupoles powered with a current of $10 \mathrm{~A}$. The reconstructed SD is shown in Fig. 5, where the blue dots are the measurements while the black line is the corresponding SD fit in Eq. (11). A good reconstruction of the stability diagram is achieved compared to the expectations.

\section{BTF MEASUREMENTS WITH SINGLE BEAM}

In the following sections, the BTF measurements will be presented and compared to the expectations by using the fitting method presented in Sec. II A. All the presented BTF measurements have been acquired on a single bunch of beam 1 at injection energy with the ADT switched off in order to avoid its component in the BTF response. In addition, we assume the impedance to be low due to the relaxed collimator settings at injection energy, and we therefore neglect its contribution in the BTF response.

\section{A. Landau octupole scan}

In the LHC, the Landau octupoles are operationally used to stabilize the beam by means of Landau damping mechanisms. The Landau octupoles introduce an amplitude detuning that is linear with the particle amplitude in the beam [2]. The powering current is related with the octupole strength and, therefore, with the tune spread provided by the Landau octupoles [2]. An octupole current scan has been performed at injection energy on a single bunch with intensity of $1.1 \times 10^{11} \mathrm{p} /$ bunch at collision tunes $\left(Q_{x} \sim 64.31, Q_{y} \sim 59.32\right)$. A BTF measurement has been acquired for each octupole current: $0,6.5,13$, and $26 \mathrm{~A}$ in both the horizontal and vertical planes of beam 1. Figure 6 shows the measured amplitude and phase responses for the different octupole strengths. As the octupole current increases, and, therefore, the tune spread in the beams, the width of the amplitude response is enlarged and the slope of the phase response decreases. For the strongest octupole strength used (26 A), a larger tune spread is observed in the horizontal plane [Fig. 6(a)] with respect to the vertical plane [Fig. 6(b)]. This was not expected, since the linear detuning with amplitude given only by the octupole magnets should have been symmetric in the two planes (assuming the same emittance, as during the experiment). The application of the fitting method Eq. (11) discussed in Sec. II A allows a quantitative comparison with the expectations. Figure 7 shows the measured phase scale in the horizontal plane (blue dots) and in the vertical plane (red dots) as a function of the octupole current. For this analysis, the measurements are compared to the model (black line) evaluated with respect to the reference case of an octupole current of $6.5 \mathrm{~A}$, for which no asymmetry in the two transverse planes has been observed, as expected from the models and for direct comparison with the operational octupole settings at the time of the measurements. 

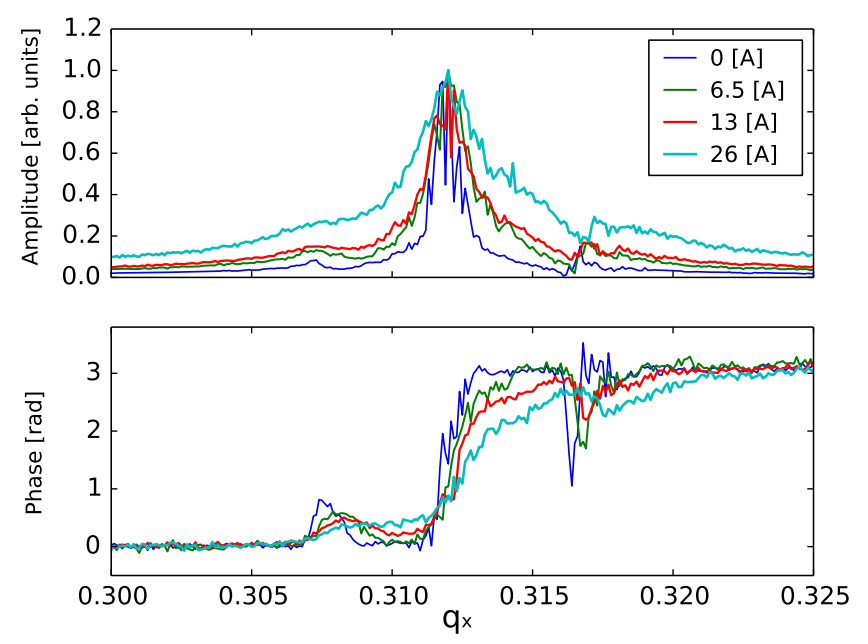

(a)
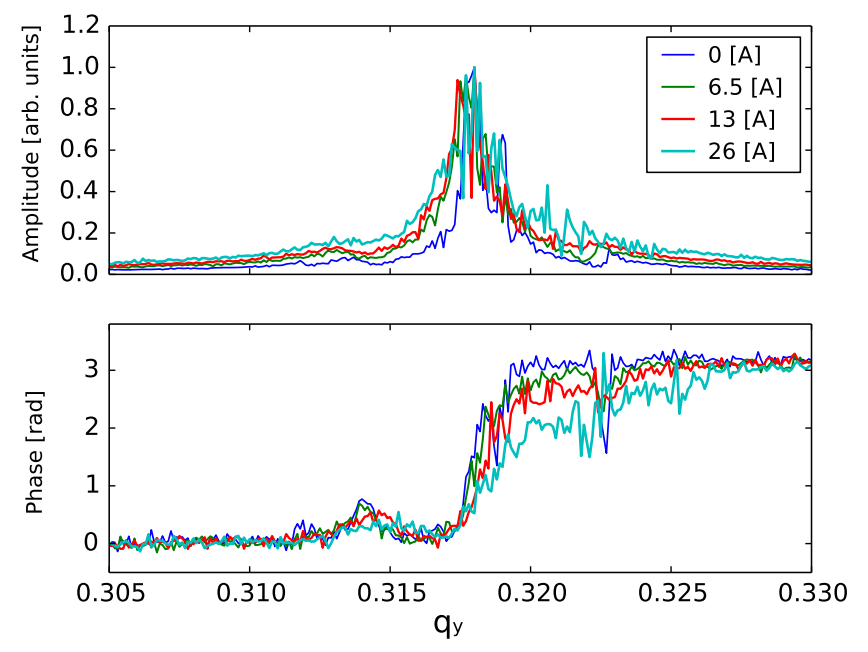

(b)

FIG. 6. Measured BTF amplitude and phase responses for beam 1 for different octupole currents at injection energy. (a) Horizontal beam response. (b) Vertical beam response.

Therefore, for such a current, the corresponding phase scale for the model is equal to 1 (black line). However, any octupole strength can be used as a reference for the model under the assumption that the tune spread is mostly provided by the octupole magnets and no other nonlinear effects are present. A larger tune spread is measured in both planes with respect to the model. The initial measured tune spread at 0 A corresponds to a tune spread parameter $\phi_{\text {scale }} \approx 0.85$ in both planes, that corresponds to a powering current of $\approx 5.5 \mathrm{~A}$. This initial nonzero value is due to the presence of other machine nonlinearities, and it is consistent with expectations at the injection energy in 2015 [26]. The red shadow represents the model expectations taking into account the nonzero initial tune spread at $0 \mathrm{~A}$ and an uncertainty of $\pm 10 \%$ on the measured beam emittance. As expected, the error on the phase scale linearly

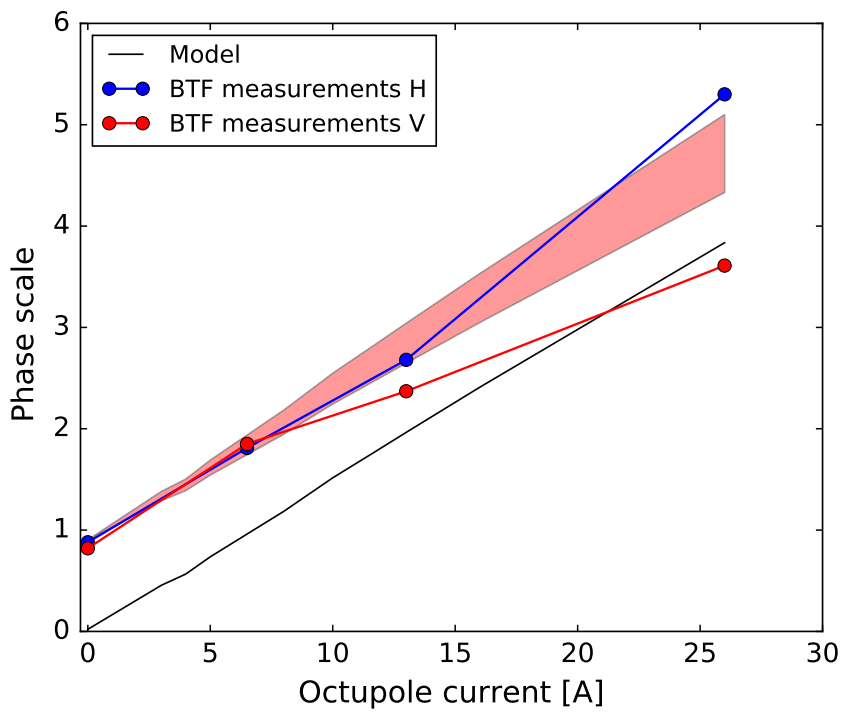

FIG. 7. The phase scale parameter evaluated from the fitting function applied to the BTF measurements at injection energy as a function of the octupole current. The blue dots represent the BTF measurements in the horizontal plane, and the red dots are the measurements in the vertical plane. The solid black line represents the expectations from the model with respect to a current $I_{\text {oct }}=6.5 \mathrm{~A}$. The model has been evaluated for an octupole current of $I_{\text {oct }}=6.5 \mathrm{~A}$ for which no asymmetry in the two transverse planes has been observed and for direct comparison with the operational octupole settings at the time of the measurements. As expected for such a current, the phase scale of the model is equal to one. The red shadow is given by the model expectations including the initial nonzero tune spread corresponding to $\approx 5.5 \mathrm{~A}$ and considering an uncertainty of $\pm 10 \%$ on the measured emittance.

increases (or decreases), since the detuning with amplitude provided by the octupoles is proportional to the octupole current $I_{\text {oct }}$ through the integrated octupole strength given in Eq. (10). The expected linear dependency with the octupole current is observed also in the measurements. However, in the vertical plane, the measured tune spread defers from the linear trend for currents above $13 \mathrm{~A}$, and it is smaller than the measured one in the horizontal plane. During the experiment, particle losses have been observed linked to the changes of the octupole current. This is visible in Fig. 8, where beam loss monitor (BLM) [27] losses at the primary collimators (TCPs) in interaction region (IR) 7 [28] are depicted as a function of the time together with the octupole current changes (the dashed red line). The blue line represents the losses at the horizontal primary collimator, the green line at the skew primary collimator, and the yellow line at the vertical collimator. The particle losses are linked to a reduction of the dynamic aperture due to the high octupole current at injection with collision tunes. The results from particle tracking simulations carried out by using the SixTrack code [29] are presented in Fig. 9. A complete lattice description is included except multipolar 


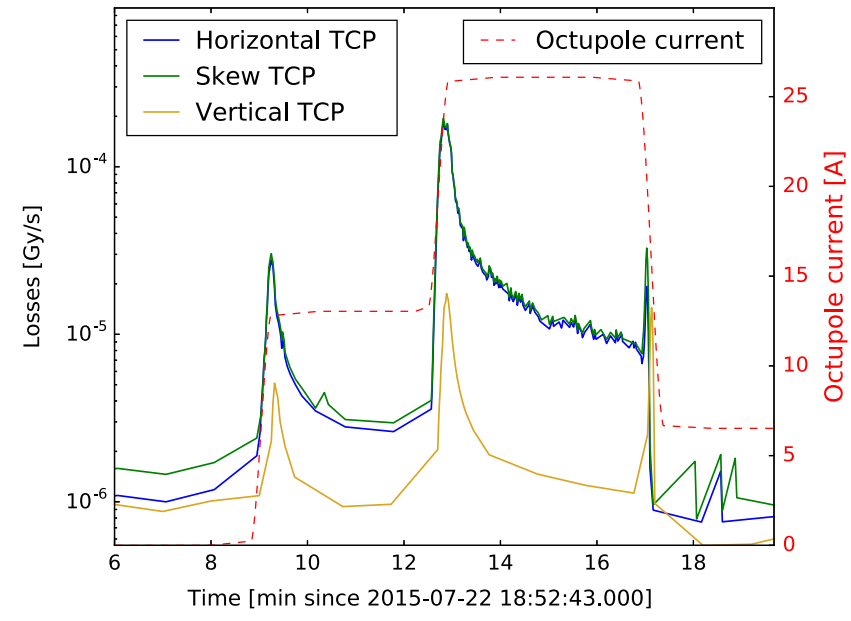

FIG. 8. Particle losses detected by the BLMs at the primary collimators in IR7 as a function of time while changing the octupole current (the dashed red line).

errors, since the nonlinearities account for roughly less than 5 A equivalent octupole current, and, therefore, for currents of 13-26 A the dominant source of tune spread affecting the beam dynamics is the octupole magnets only.

The particle distribution is tracked over $10^{6}$ turns. Figure 9 shows the surviving particle ratio defined as

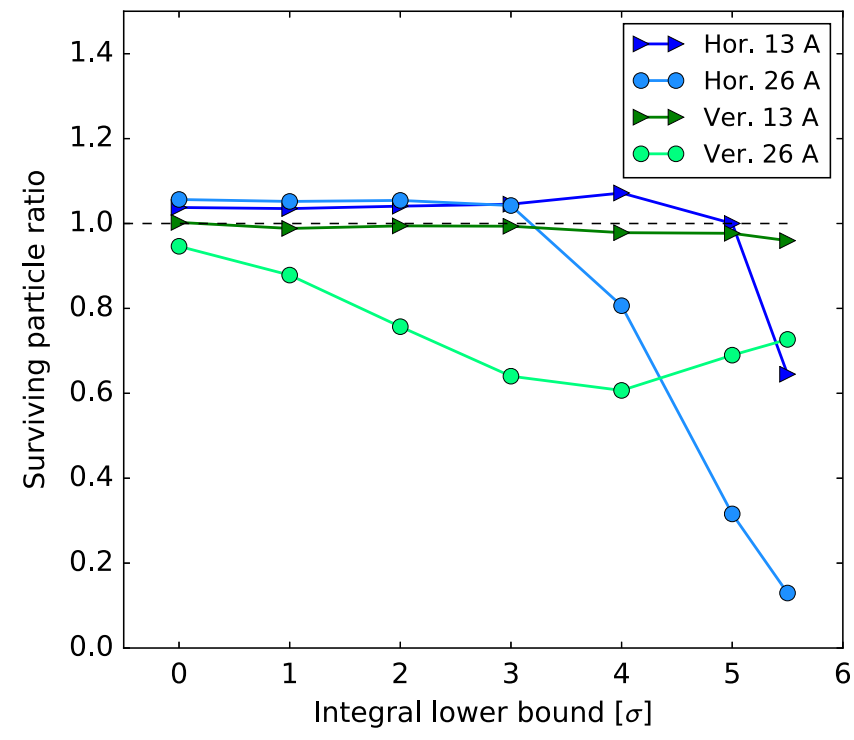

FIG. 9. Final over initial particle ratio calculated as the ratio of the integral of the final tracked distribution (Gaussian) after $10^{6}$ turns, over the integral of the initial distribution, as a function of the lower bound (expressed in units of transverse rms beam size $\sigma$ ) used for the calculation of the integral. The upper bounds of the particle distribution integrals are fixed to $6 \sigma$. The dark blue line with dark blue triangles and the light blue dotted line represent the horizontal plane for an octupole current of 13 and $26 \mathrm{~A}$, respectively. The dark green line with dark green triangles and the light green dotted line represent the vertical plane for an octupole current of 13 and $26 \mathrm{~A}$, respectively. the ratio of the integral of the final distribution (Gaussian) over the integral of the initial distribution, as a function of the lower bound (expressed in units of transverse rms beam size $\sigma$ ) used for the evaluation of the integral. The upper bounds of the particle distribution integrals are fixed to $6 \sigma$. As visible, the ratio shows that, while increasing the octupole current, particles are lost more and more from the tails but also from the beam core for higher octupole current. Particle losses occur in both planes with different amplitudes affected. The increasing loss rates are in agreement with the observation during the experiment at the LHC. In particular, for the case with an octupole current of $26 \mathrm{~A}$, a reduction up to $40 \%$ is observed in the beam core for the vertical plane (light green dotted line). This would explain the reduction of the measured tune spread in the BTF for such current. Indeed, the particles that mostly
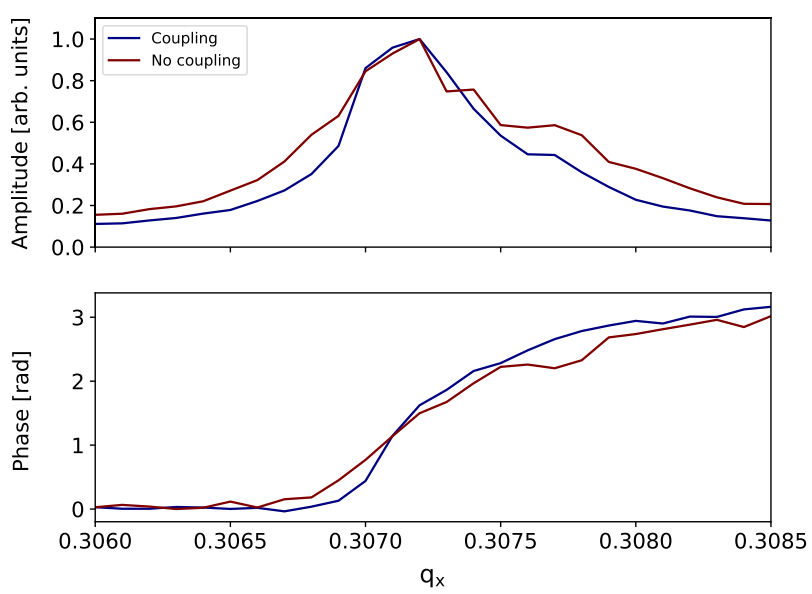

(a)
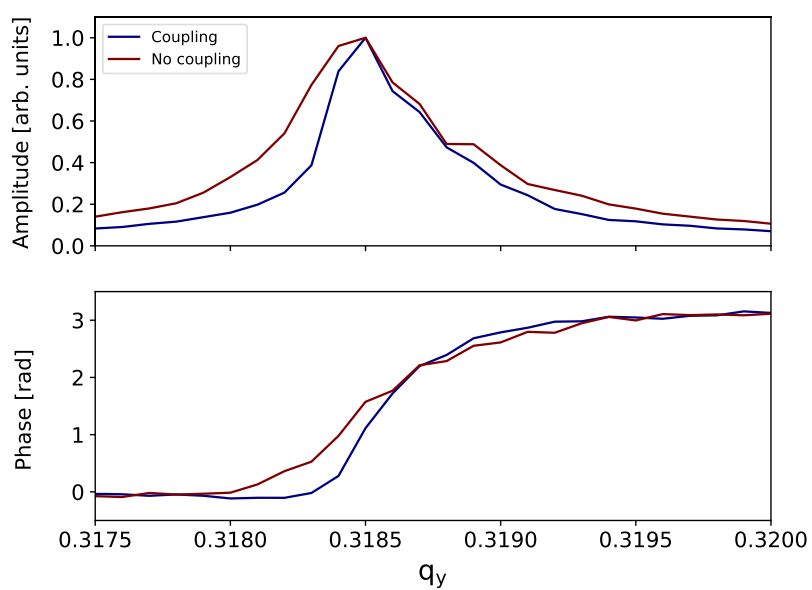

(b)

FIG. 10. Simulated BTF by using the COMBI code in the presence of linear coupling (the blue line) with a global coupling coefficient $\left|C^{-}\right| \approx 0.007$ and without linear coupling (the red line). Simulations have been carried out for an octupole current of 4 A. (a) Simulated BTF response in the horizontal plane. (b) Simulated BTF response in the vertical plane. 


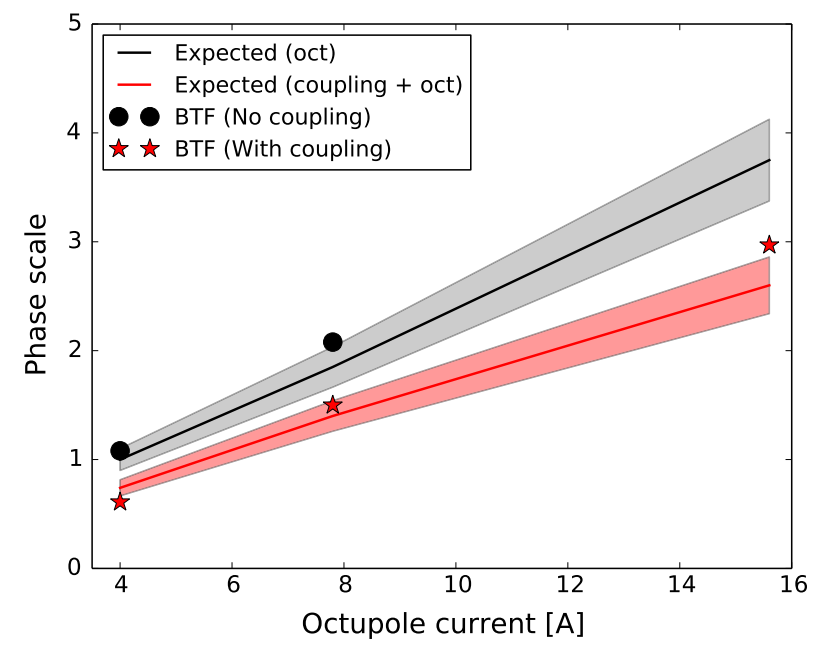

(a)

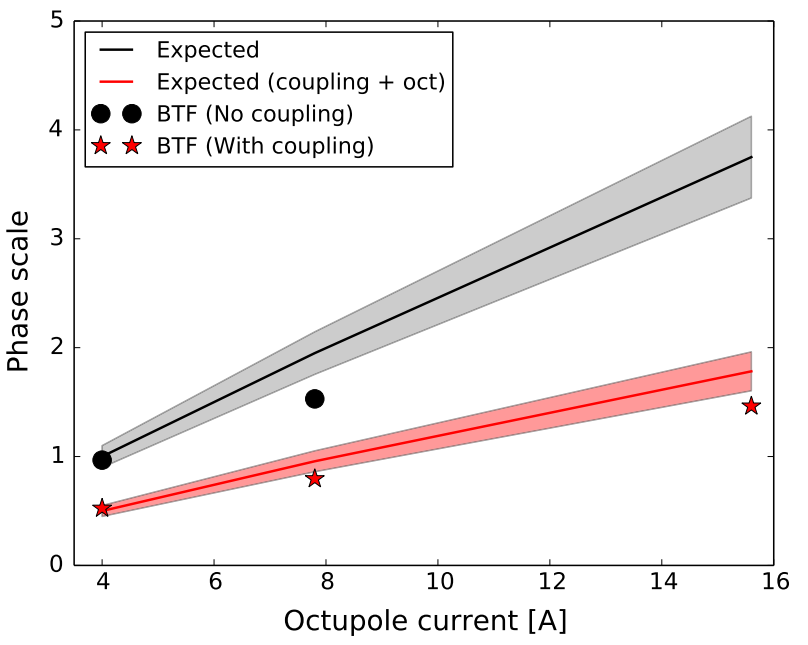

(b)

FIG. 11. Measured phase scale parameter evaluated by using the fitting method with respect to the analytical reference case of 4 A without linear coupling. The black dots are the measurements without linear coupling, while the red stars are the measurements with $\left|C^{-}\right|=0.006$. The red and the black shadows represent the analytical expectations considering an uncertainty of $\pm 10 \%$ on the measured emittance for the case with and without linear coupling, respectively. (a) Phase scale parameters measured in the horizontal plane.

(b) Phase scale parameters measured in the vertical plane.

contribute to the BTF response are those in the beam core and not the ones in the tails [30]. Particle losses in the core of the beam reduce the Landau damping expected for an unperturbed Gaussian distribution. Therefore, increasing the tune spread is not beneficial for Landau damping if particle losses are present.

\section{B. Effects of linear coupling on BTF response}

The transverse linear coupling might cause destabilizing effects [31-33] due to a reduction of the Landau damping of the beams. The effects of the linear coupling resonance on the tune spread of the beams have been measured and quantified by the BTF measurements. As an example, the simulated BTF response computed by the COMBI code [22] in the presence of transverse linear coupling is shown in Fig. 10 (the blue line) and compared to the case without linear coupling (the red line) both in the horizontal [Fig. 10(a)] and in the vertical [Fig. 10(b)] plane. In the simulations, a global coupling coefficient $\left|C^{-}\right|$(defined as the closest-tune approach) $\approx 0.007$ [34-36] has been included. This extreme (but not impossible) value has been considered here for a better visibility by the naked eye of the effect of the linear coupling on the BTF response presented in Fig. 10. As visible, in the presence of transverse linear coupling, the tune spread, and therefore the Landau damping, is reduced in both planes (reduced amplitude width and increased phase slope in the BTF response). The BTF response becomes asymmetric in the two planes with a smaller tune spread in the vertical plane than the horizontal plane due to coupling resonance in the tune diagram $[32,33,37]$. The modification of Landau damping in the presence of transverse linear coupling has been experimentally investigated for the first time by means of BTF measurements in the LHC. During the experiment, the global linear coupling was corrected and introduced in a controlled way for quantitative comparisons with expectations. The $\left|C^{-}\right|$value has been evaluated by the minimum tune approach [36]. With a fixed value of $\left|C^{-}\right|=0.006$, an octupole current scan was performed at injection energy: The octupole strength was varied from a current of 4 to $15.6 \mathrm{~A}$. BTF measurements were acquired at each octupole current change on a single bunch of beam 1 . The results of the measurements are shown in Fig. 11. The measured phase scale parameters evaluated by using the fitting method [Eq. (11)] are plotted as a function of the octupole current and compared to the models. The red solid line represents the model expectations evaluated by using the PySSD code and including both octupoles and linear coupling in the MAD-X lattice [38] in order to evaluate the phase scale using the fitting method. The black solid line includes only the octupoles in the MAD-X model. The red and the black shadows include in the models an uncertainty of $\pm 10 \%$ on the measured emittance value. The black dots are the measurements without linear coupling, while the red stars represent the measurements in the presence of the linear coupling $\left(\left|C^{-}\right|=0.006\right)$ both in the horizontal plane [Fig. 11(a)] and in the vertical plane [Fig. 11(b)]. An overall reduction of the tune spread is measured in both planes. However, the tune spread reduction is more important in the vertical plane as expected from the simulated BTF (Fig. 11), and the beam response is asymmetric between the two planes. For the largest octupole current of $15.6 \mathrm{~A}$, the tune spread is reduced by a factor of 2 with respect to the horizontal plane, and the experimental data reproduce well the expectations in both cases. 


\section{CONCLUSIONS}

The summary of the BTF measurements acquired on a single beam has been presented in this article. Because of the fact that the height of the BTF amplitude response depends not only on the calibration factor of the BTF system itself, but also on the tune spread in the beams, it can be expressed only in arbitrary units. As a consequence, the extrapolated SD from BTF measurements has not the correct size in the complex plane of the head-tail coherent tune shifts. To overcome this problem, the development of a fitting method was necessary in order to compare the measurements with the model expectations. The developed fitting method has been described and applied to measurements in the LHC. In order to prove the reliability of the method, it was first applied to simulations including the well-known case of linear detuning with amplitude provided by the Landau octupoles. A full agreement with the analytical expectations was found. The fitting method was then applied to the measured BTF responses in order to probe the Landau damping in the beams. The BTF response has been measured at injection energy for various Landau octupole strengths and compared to the expectations by applying the fitting method. As expected, a linear trend as a function of the octupole strength has been observed in the BTF measurements, and a good agreement with expectations has been found. Because of particle losses observed for high octupole currents, the phase scale in the vertical plane resulted to be reduced with respect to the analytical expectations computed for a Gaussian particle distribution in the beam. It was observed for the first time that, in the presence of particle losses, increasing the tune spread is not beneficial for Landau damping as much as expected for the Gaussian case. Since the linear coupling might modify the beam stability, the Landau damping of the beams has been probed through BTF measurements in the presence of transverse linear coupling for various Landau octupole strengths. The destabilizing effects of linear coupling have been quantified by the BTF measurements and compared to the models. The BTF measurements agree with the predictions and the expected trend has been reproduced: In the presence of linear coupling, an overall reduction of the Landau damping of the beams has been measured in both planes with a more important reduction in the vertical plane. The tune spread in the vertical plane results to be less than the horizontal plane by almost a factor of 2 for the largest octupole strength used.

The fitting function method provides a quantitative analysis and allows comparisons of the measured BTF with the present models used to predict the beam stability in the LHC. It has been shown that in the case of a single beam at injection energy it is a reliable method for the investigation of the transverse beam stability. However, the BTF system and fitting method present some limitations: (i) It relies on an approximation of the tune spread when unknown nonlinear effects are present and of the beam distribution that is considered as a Gaussian particle distribution in the models [30]; (ii) the effect of the chromaticity in the BTF response causes distortion in the reconstruction of the stability diagrams [30]; (iii) when the impedance is strong, the BTF response does not allow one to apply the fitting method to extrapolate the tune spread from the measurements.

\section{ACKNOWLEDGMENTS}

The presented studies have been supported by the Swiss Accelerator Research and Technology (CHART) institute. We thank the BI team for the development of the BTF system, the OP team for helping during the data taking, and W. Herr, N. Mounet, T. H. Persson, R. Tomás, E. H. Maclean, and G. Trad for fruitful discussions.

[1] L. Landau, On the vibrations of the electronic plasma, J. Phys. USSR 10, 445 (1965).

[2] J. Gareyte et al., Landau Damping, Dynamic Aperture and Octupoles in LHC, LHC Project Report 91, CERN, Geneva, Switzerland.

[3] X. Buffat, W. Herr, N. Mounet, T. Pieloni, and S. White, Stability diagrams of colliding beams in the Large Hadron Collider, Phys. Rev. Accel. Beams 17, 111002.

[4] D. Mohl and A. M. Sessler, The use of RF-knockout for determination of the characteristics of the transverse coherent instability of an intense beam, eConf C710920, 334 (1971), https://inspirehep.net/literature/73329.

[5] A. W. Chao, Physics of Collective Beams Instabilities in High Energy Accelerators, Wiley series on Beam Physics and Accelerator Technology (Wiley, New York, 1993).

[6] J. Borer, G. Guignard, A. Hofmann, E. Peschardt, F. Sacherer, and B. Zotter, Information from beam response to longitudinal and transverse excitation, IEEE Trans. Nucl. Sci. 26, 3405 (1979).

[7] J.S. Berg and F. Ruggiero, Landau damping with twodimensional betatron tune spread, Technical Report No. CERN SL-AP-96-71, 1996.

[8] M. G. Minty and F. Zimmermann, Measurement and Control of Charged Particle Beams (Springer, New York, 2003).

[9] P. Chou and G. Jackson, Beam transfer function and transverse impedance measurements in the Fermilab Main Ring, in Proceedings of the Particle Accelerator Conference, Dallas, TX, 1995 (IEEE, New York, 1995), Vol. 5.

[10] H. Damerau et al., Evaluation of the broadband longitudinal impedance of the CERN PS, Report No. CERNATS-2012-064 MD, CERN, 2012.

[11] P. Gorgen, O. Boine-Frankenheim, and W. Fischer, Beam transfer functions for relativistic proton bunches with beam-beam interaction, Nucl. Instrum. Methods Phys. Res., Sect. A 777, 43 (2015).

[12] J.-Y. Hemery, A. Hofmann, J-P. Koutchouk, S. Myers, and L. Vos, Investigation of the coherent beam-beam effects in the ISR, IEEE Trans. Nucl. Sci. 28, 2497 (1981). 
[13] A. Hofmann, The beam-beam transfer function, ISR Performance Report, CERN, 1981.

[14] X. Buffat, Transverse beams stability studies at the Large Hadron Collider, Ph.D. thesis, Ecole Polytechnique Federale de Lausanne (EPFL), 2015.

[15] C. Tambasco et al., First beam transfer function measurements in the LHC, Technical Report No. CERN-ACCNOTE-2016-0012, CERN, Geneva, Switzerland, 2016.

[16] C. Tambasco et al., MD 382: Beam transfer function and diffusion mechanisms, Technical Report No. CERN-ACCNOTE-2016-0016, CERN, 2016.

[17] C. Tambasco et al., MD 1407-Landau damping: Beam transfer functions and diffusion mechanisms, Technical Report No. CERN-ACC-NOTE-2017-0026, CERN, Geneva, Switzerland, 2017.

[18] C. Tambasco et al., MD 1856-Landau damping: Beam transfer functions and diffusion mechanisms, Technical Report No. CERN-ACC-NOTE-2017-0027, CERN, Geneva, Switzerland, 2017.

[19] C. Tambasco et al., MD 2722: Investigation of Landau damping by means of BTF measurements, Technical Report No. CERN-ACC-NOTE-2018-0016, CERN, Geneva, Switzerland, 2018.

[20] C. Tambasco et al., MD 2197: Experimental studies of Landau damping by means of beam transfer function measurements in the presence of beam-beam interactions and diffusive mechanisms, Technical Report No. CERNACC-NOTE-2018-0017, CERN, Geneva, Switzerland, 2018.

[21] M. Gasior and R. Jones, High sensitivity tune measurement by direct diode detection, Report No. CERN-AB-2005-060 BDI, 2005.

[22] T. Pieloni and W. Herr, Models to study multi bunch coupling through head-on and long-range beam-beam interactions, CERN LHC Project Report No. 937 and Proceedings of the 10th European Particle Accelerator Conference, Edinburgh, Scotland, 2006 (EPS-AG, Edinburgh, Scotland, 2006).

[23] T. Pieloni and W. Herr, Coherent beam-beam in the CERN large hadron collider (LHC) for multiple bunches, different collision schemes and machine symmetries, in Proceedings of the 21st Particle Accelerator Conference, Knoxville, TN, 2005 (IEEE, Piscataway, NJ, 2005).

[24] T. Pieloni, A study of beam-beam effects in hadron colliders with a large number of bunches, Ph.D. thesis, Ecole Polytechnique Federale de Lausanne (EPFL), 2008.
[25] W. Herr and T. Pieloni, https://twiki.cern.ch/twiki/bin/ view/ ABPComputing/COMBI.

[26] R. Tomás, Collecting amplitude detuning measurements from 2012, Technical Report No. CERN-ACC-NOTE2014-0027, CERN, 2014.

[27] B. Dehning et al., The LHC beam loss measurement system, in Proceedings of the 22nd Particle Accelerator Conference, PAC-2007, Albuquerque, NM (IEEE, New York, 2007).

[28] R. Bruce et al., Simulations and measurements of beam loss patterns at the CERN Large Hadron Collider, Phys. Rev. Accel. Beams 17, 081004 (2014).

[29] F. Schmidt, SixTrack user's reference manual, Technical Report, CERN, 1994.

[30] C. Tambasco, Beam transfer function measurements and transverse beam stability studies for the Large Hadron Collider and its high luminosity upgrade, Ph.D. thesis, Ecole Polytechnique Federale de Lausanne (EPFL), 2017.

[31] E. Métral, Coupled Landau damping of transverse coherent instabilities in particle accelerators, Ph.D. thesis, Université Joseph Fourier, 1999.

[32] E. Métral, G. Hoffstaetter, and F. Willeke, Destabilizing effect of linear coupling in the HERA proton ring, in Proceedings of the 8th European Particle Accelerator Conference, Paris, 2002 (EPS-IGA and CERN, Geneva, 2002), pp. 1535-1537.

[33] L. R. Carver, X. Buffat, K. Li, E. Métral, and M. Schenk, Transverse beam instabilities in the presence of linear coupling in the Large Hadron Collider, Phys. Rev. Accel. Beams 21, 044401 (2018).

[34] R. Tomás, M. Bai, R. Calaga, W. Fischer, A. Franchi, and G. Rumolo, Measurement of global and local resonance terms, Phys. Rev. Accel. Beams 8, 024001 (2005).

[35] A. Franchi, R. T. Garcia, and G. Vanbavinkhove, Computation of the coupling resonance driving term f1001 and the coupling coefficient $\mathrm{C}$ from turn-by-turn singleBPM data, Technical Report No. CERN-BE-Note-2010016, 2010.

[36] T. Persson and R. Tomàs, Improved control of the betatron coupling in the Large Hadron Collider, Phys. Rev. Accel. Beams 17, 051004 (2014).

[37] R. Tomas, T. Persson, and E. Maclean, Amplitude dependent closest tune approach, Phys. Rev. Accel. Beams 19, 071003 (2016).

[38] MAD-Methodical accelerator design, https://mad.web .cern.ch/mad/. 